\title{
A Novel Routing Technique for Congestion Avoidance in WSN using Bat Algorithm
}

\author{
Aditya Prakash \\ Student, Dept of ECE \\ CTIEMT, Jalandhar, Punjab
}

\author{
Noorinder Kaur \\ Asst. Professor, Dept of ECE \\ CTIEMT, Jalandhar, Punjab
}

\begin{abstract}
Nature inspired optimization algorithms are useful for solving different kind of engineering problems, combinatorial problems and many more. Bat Algorithm is one of the nature inspired techniques which fulfill the criteria of finding the optimized and better result, in solving most of the problems. Routing is one of the combinatorial optimization problems, which can be solved using Bat Algorithm. Many researchers have contributed in this field by proposing and developing one or the other techniques to solve the problem of routing. In this paper, Bat Algorithm is used to solve the same and the problem of congestion over optimal path is avoided by the proposed algorithm in wireless sensor network. Experimental results show that the hybridization of bat algorithm and congestion avoidance strategy proved to be efficient than queue based congestion avoidance strategy while solving problem at hand on the basis of mean, minimum, maximum and median values.
\end{abstract}

\section{Keywords}

Bat Algorithm; Collision; Routing; WSN

\section{INTRODUCTION}

Combinatorial Optimization deals in finding the optimize solution among finite set of solutions. It is generally employed in those application areas, where exhaustive search is unable to provide the desired solutions. Problems including Minimum Spanning Tree, Knapsack Problem, Chinese Postman Problem, Scheduling Problem, Traveling Salesman Problem and many more, can be solved using this type of optimization. The focus of this research is to study the problem of routing and finding the solutions for the same using nature inspired intelligence optimization techniques, while ensuring congestion free optimal path. The concept of routing is to find the series of routers/intermediate nodes through which packets should traverse, while minimizing the cost/distance or fulfilling the other quality of service parameters like minimizing delay, maximizing throughput, having minimum congestion. Routing can be considered as an undirected weighted graph, in which vertices are routers/nodes and edges are paths. Each node is connected with remaining other nodes. It is one of the more explored problems in optimization. Some researchers consider routing as one of the benchmarks for proving the efficiency and performance of any optimization technique. It has been implemented in logistics, planning and manufacturing of microchips, in DNA sequencing, in astronomy, as astronomers want to optimize the time spent in moving the telescope.

\section{RELATED WORK}

In [20], author has reviewed wireless sensor networks' applications, their significant QOS needs, and routing protocols which satisfy those QOS needs. In [9], based on structure of a network, routing protocols in WSN can be broadly classified in three categories: Flat routing, Hierarchal routing or Cluster based routing, and Location based routing. In [16], author figured out the challenges and security concern in WSN routing protocols, the challenges are mainly related to management of limited power of nodes, lack of resources, scalability, mobility, data aggregation, quality of services, security and many more. In [5], author has done the analysis of various BAT variants and further research areas are explored in the field of meta-heuristic approaches. In this work [19], author has discussed about popular protocol Low Energy Adaptive Clustering Hierarchy (LEACH) which is based on adaptive clustering technique. In paper [6], author has summarized the review of research related to nature inspired metaheuristic algorithms, primarily focusing on Bat Algorithm. In the paper [22], author has analyzed the various protocols which are based on swarm intelligence. In [21], author has identified that the most of the research in swarm intelligence has focused on the reverse engineering and the adaptation of collective behaviors observed in natural systems with the aim of designing effective algorithm. In [24], Swarm based routing protocols are classified into three categories: Ant based, Bee based and Slim based. In paper [23], author has related the characteristics of bat with routing technique. In the paper [3], Opportunistic routing protocols (ORP) uses the broadcasting nature of wireless sensor network. In order to achieve higher energy efficiency author has proposed in paper [4], the usage of swarm intelligence, which observes the collective behavior of social insects and other animal societies. In paper [2], author has discussed a technique to handle the congestion and find the optimal path to reach destination in vehicular adhoc network, using bat algorithm. In paper [17], author has proposed the use of genetic algorithm in wireless sensor network to achieve the efficient routing. The author stated in [25], classification of WSN networks has been done as per network requirements such as limited energy availability, low memory and reduced processing power. In paper [10], author has introduced the "guidable bat algorithm" and validated the result using Rastrigin and Griewangk function. The same has been achieved by modifying velocity and frequency using Doppler Effect, replacing low pass filter to filter noise. By using transfer function to map continuous search space to binary search space, author has proposed the Binary bat algorithm in [15] and verified with optical buffer design in optical engineering. To increase the exploration and local search capability, author has used Invasive weed optimization in [8] and formulated the new algorithm Enhanced Bat algorithm and validated the result with spiral design, pressure vessel design and Welded beam. In the paper [1] three modules have been proposed; prediction of destination location, formation of region and selection of optimized route. In the paper [18], author has worked to enhance the bat algorithm and proposed multiobjective bat algorithm and validated the results using 
unimodal and multimodal functions. In paper [13], work has been done towards addition of mutation and crossover to avoid trapping in local optima and development of new hybridized bat algorithm with differential evolution. In the paper [14], author has introduced chaotic bat algorithm and worked on parameter initialization using chaotic sequences rather than random initialization and validated the result using high dimensional and low dimensional functions. In paper [11], author has used the concept of parameter initialization but with the help of fuzzy logic and verified the result by providing optimum solution to inverted pendulum problem. In [12], author has chosen RLF algorithm for initialization and sigmoid function to represent continuous space in binary encoding, to solve the problem of graph coloring. By enhancing exploration and exploitation, dynamic virtual bat algorithm is developed by the author in [17] and results are verified using unimodal and multimodal functions. In [26], author has selected the optimal route based on the queue size available with all the nodes present in the network. The motive of this research is to select that optimal node, which is having maximum value of the queue. For the same, author has compared the results with CODA, PSO and ACO based congestion route optimization algorithms. To develop an efficient routing algorithm, one should consider the other factors as well, primarily congestion on the optimal route. In this work, the focus is to develop such an algorithm which will not only find the optimal solution but also takes care of the congestion problem.

\section{PROBLEM FORMULATION}

To solve routing problem and minimize energy consumption, various optimization techniques are preferred by researchers.

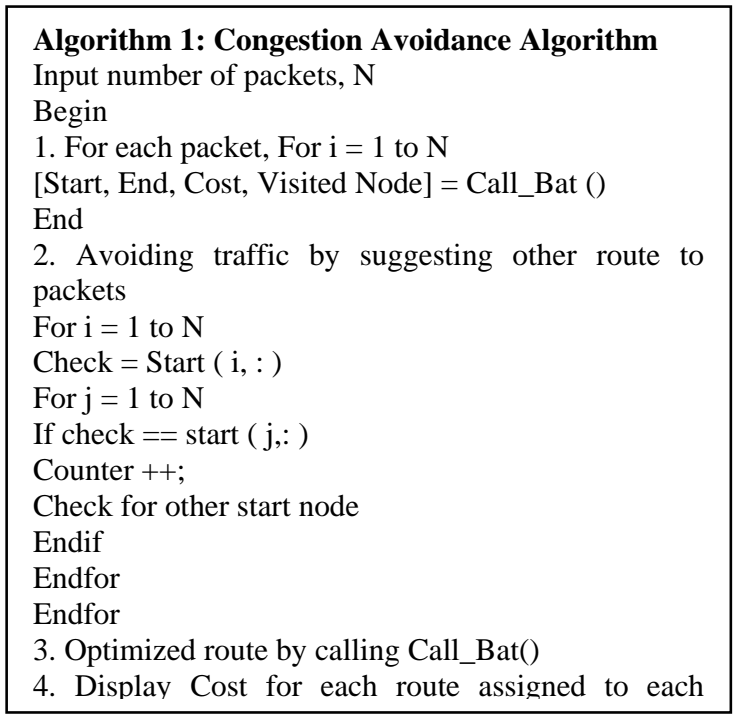

Figure 1: Pseudocode of Traffic Avoidance using Bat Algorithm

Node deployment, energy efficiency, congestion and network lifetime are the main challenges of wireless sensor networks which can be overcome using optimization techniques. Depending upon the nature of algorithm and desired solution the application of Genetic algorithm, PSO, Bat algorithm and other nature inspired algorithms are introduced. The drawback of [26] algorithm is that due to flooding of control information related to queue size, consumes the available bandwidth to some extent. Moreover, delay is also introduced with the above mentioned approach. This work is proposed to tackle the situation, which is mentioned above. The focus is to ensure that no such delay incur or to reduce the delay. Another aspect of this research work is to reduce the control packets, specifying the size of queue, in the network.

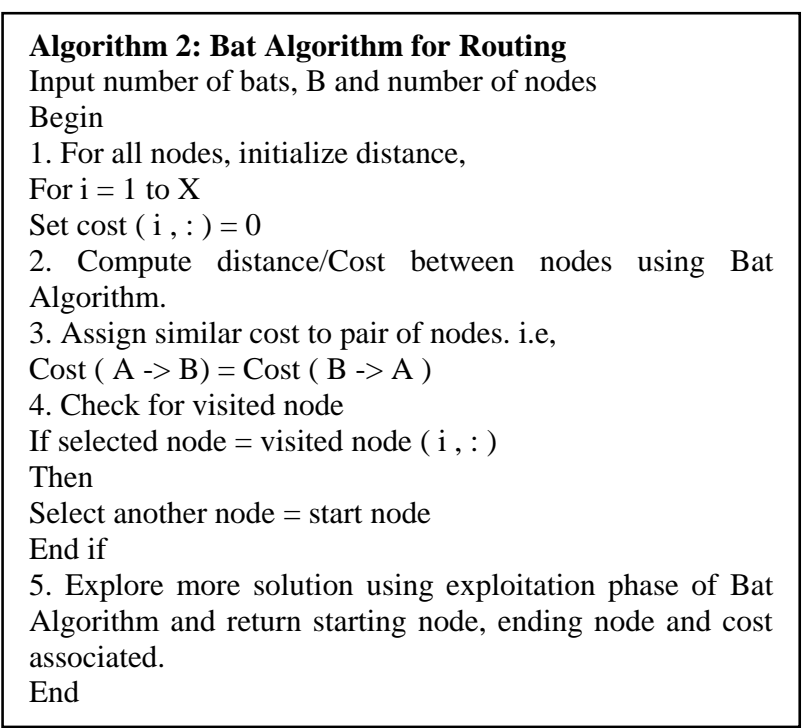

Figure 2: Pseudocode of Routing using Bat Algorithm

\section{RESULTS \& DISCUSSIONS}

The proposed work is implemented in MatLab. While implementing the proposed algorithm, the values of number of packets and number of nodes are varied to evaluate the performance of proposed algorithm. It has been observed that in search of optimal route, the time taken, by packets, will increase as the number of nodes increase.

Table 1: Time comparison of proposed work vs. base paper work for 10 Packets to explore different nodes

\begin{tabular}{|c|c|c|c|c|c|c|}
\hline \multicolumn{6}{|c|}{ Time comparison of Standard vs Proposed Routing Algorithm } \\
\hline \multirow{2}{*}{$\begin{array}{c}\text { Time (in } \\
\text { Seconds) }\end{array}$} & $\begin{array}{c}\text { Nodes- 10, } \\
\text { Packets-10 }\end{array}$ & \multicolumn{2}{c|}{$\begin{array}{c}\text { Nodes- 15, } \\
\text { Packets-10 }\end{array}$} & \multicolumn{2}{c|}{$\begin{array}{c}\text { Nodes- 20, } \\
\text { Packets-10 }\end{array}$} \\
\cline { 2 - 7 } & $\begin{array}{c}\text { tandard } \\
\text { work }\end{array}$ & $\begin{array}{c}\text { Proposed } \\
\text { work }\end{array}$ & $\begin{array}{c}\text { Standard } \\
\text { work }\end{array}$ & $\begin{array}{c}\text { Proposed } \\
\text { work }\end{array}$ & $\begin{array}{c}\text { Standard } \\
\text { work }\end{array}$ & $\begin{array}{c}\text { Proposed } \\
\text { work }\end{array}$ \\
\hline Best & 2.13 & 1.97 & 12.50 & 12.29 & 48.56 & 39.12 \\
\hline Median & 2.30 & 2.01 & 13.08 & 12.53 & 49.16 & 46.35 \\
\hline Worst & 2.73 & 2.18 & 13.78 & 14.02 & 54.03 & 56.16 \\
\hline Mean & 2.32 & 2.05 & 13.16 & 12.73 & 49.70 & 46.70 \\
\hline
\end{tabular}

As discussed earlier to avoid congestion, threshold value is set for usability of proper path. If usability factor attains its maximum to threshold value, the packets will be re-routed over new path. As the number of same source node increases, it is quite natural that packets try to use the same optimal path to minimize the cost but at the same time, there is a need to avoid the congestion over same path by rerouting the packets which make the network more efficient. 
Table 2: Time comparison of proposed work vs. base paper work for 15 Packets to explore different nodes

\begin{tabular}{|c|c|c|c|c|c|c|}
\hline \multicolumn{6}{|c|}{ Time comparison of Standard vs Proposed Routing Algorithm } \\
\hline \multirow{2}{*}{$\begin{array}{c}\text { Time (in } \\
\text { Seconds) }\end{array}$} & $\begin{array}{c}\text { Nodes- 10, } \\
\text { Packets-15 }\end{array}$ & $\begin{array}{c}\text { Nodes- 15, } \\
\text { Packets-15 }\end{array}$ & \multicolumn{2}{c|}{$\begin{array}{c}\text { Nodes- 20, } \\
\text { Packets-15 }\end{array}$} \\
\cline { 2 - 7 } & $\begin{array}{c}\text { tandard } \\
\text { work }\end{array}$ & $\begin{array}{c}\text { Proposed } \\
\text { work }\end{array}$ & $\begin{array}{c}\text { Standard } \\
\text { work }\end{array}$ & $\begin{array}{c}\text { Proposed } \\
\text { work }\end{array}$ & $\begin{array}{c}\text { Standard } \\
\text { work }\end{array}$ & $\begin{array}{c}\text { Proposed } \\
\text { work }\end{array}$ \\
\hline Best & 3.36 & 3.02 & 18.87 & 17.12 & 71.92 & 69.88 \\
\hline Median & 3.42 & 3.16 & 19.64 & 18.06 & 73.53 & 71.25 \\
\hline Worst & 3.56 & 3.45 & 20.05 & 23.07 & 74.52 & 78.58 \\
\hline Mean & 3.43 & 3.17 & 19.57 & 19.15 & 73.36 & 72.01 \\
\hline
\end{tabular}

Table 1 and 2 depicts the improvement in the result of proposed work on the basis of best, worst, mean and median for fixed number of packets 10 for varying count of nodes $[10,15,20]$.

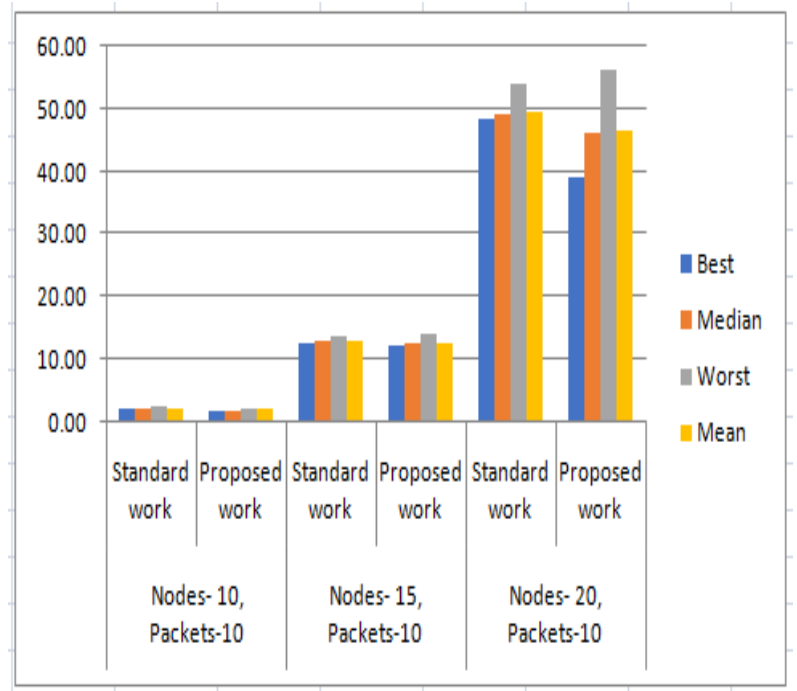

Figure 3: Graphical Representation of Comparison of Standard vs. Proposed Work, considering Time as factor for 10 packets

Considering the time taken by standard algorithm used for congestion avoidance and time taken by proposed algorithm, to obtain optimal results, Figure 3 and Figure 4 represents the graphical form of data as mentioned in table 1 and table 2. As it is clearly visible, as the count of nodes increase, it will lead to great change in the time interval to find optimal route.

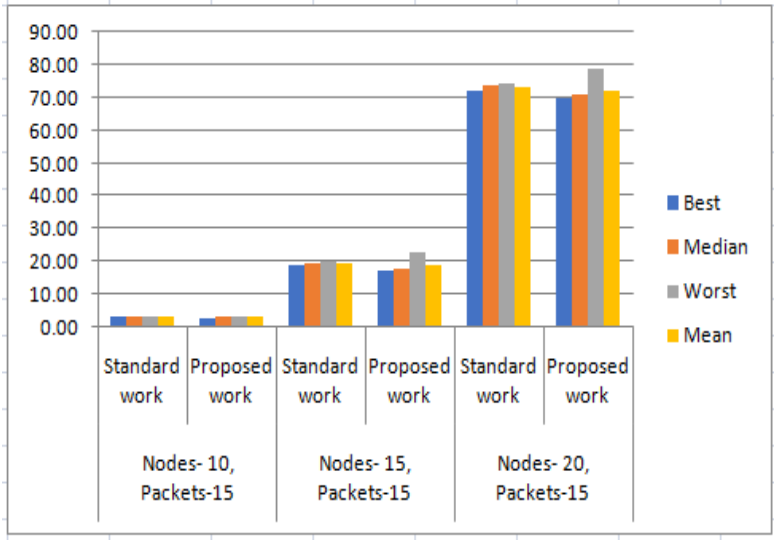

Figure 4: Graphical Representation of Comparison of Standard vs. Proposed Work, considering Time as factor for 15 packets

In Table 3 and 4, it has been observed that in search of optimal route the cost will increase as the number of nodes increase. As bats have to explore more number of optimal nodes, which will lead to the increase in cost factor. However, the cost reflecting is minimal, as the optimal path is traced by nature inspired algorithm. Comparison shows the better result of proposed algorithm.

Table 3: Cost comparison of proposed work vs. base paper work for 10 Packets to explore different nodes

\begin{tabular}{|c|c|c|c|c|c|c|}
\hline \multicolumn{5}{|c|}{ Cost comparison of Standard vs Proposed Routing Algorithm } \\
\hline \multirow{2}{*}{$\begin{array}{c}\text { Cost } \\
\text { Factor }\end{array}$} & $\begin{array}{c}\text { Nodes- 10, } \\
\text { Packets-10 }\end{array}$ & \multicolumn{2}{c|}{$\begin{array}{c}\text { Nodes- 15, } \\
\text { Packets-10 }\end{array}$} & \multicolumn{2}{c|}{$\begin{array}{c}\text { Nodes- 20, } \\
\text { Packets-10 }\end{array}$} \\
\cline { 2 - 7 } & $\begin{array}{c}\text { Standard } \\
\text { work }\end{array}$ & $\begin{array}{c}\text { Proposed } \\
\text { work }\end{array}$ & $\begin{array}{c}\text { Standard } \\
\text { work }\end{array}$ & $\begin{array}{c}\text { Proposed } \\
\text { work }\end{array}$ & $\begin{array}{c}\text { Standard } \\
\text { work }\end{array}$ & $\begin{array}{c}\text { Proposed } \\
\text { work }\end{array}$ \\
\hline Best & 250.35 & 143.65 & 243.05 & 186.79 & 239.94 & 240.83 \\
\hline Median & 280.24 & 156.80 & 287.72 & 216.87 & 276.56 & 267.69 \\
\hline Worst & 305.93 & 176.70 & 348.85 & 293.48 & 481.26 & 365.67 \\
\hline Mean & 280.39 & 158.36 & 288.97 & 220.34 & 293.18 & 291.08 \\
\hline
\end{tabular}

Table 4: Cost comparison of proposed work vs. base paper work for 15 Packets to explore different nodes

\begin{tabular}{|c|c|c|c|c|c|c|}
\hline \multicolumn{6}{|c|}{ Cost comparison of Proposed work Vs Base Paper work } \\
\hline $\begin{array}{c}\text { Cost } \\
\text { Factor }\end{array}$ & $\begin{array}{c}\text { Nodes- 10, } \\
\text { Packets-15 }\end{array}$ & $\begin{array}{c}\text { Nodes- 15, } \\
\text { Packets-15 }\end{array}$ & \multicolumn{2}{c|}{$\begin{array}{c}\text { Nodes- 20, } \\
\text { Packets-15 }\end{array}$} \\
\cline { 2 - 7 } & $\begin{array}{c}\text { Standard } \\
\text { work }\end{array}$ & $\begin{array}{c}\text { Proposed } \\
\text { work }\end{array}$ & $\begin{array}{c}\text { Standard } \\
\text { work }\end{array}$ & $\begin{array}{c}\text { Proposed } \\
\text { work }\end{array}$ & $\begin{array}{c}\text { Standard } \\
\text { work }\end{array}$ & $\begin{array}{c}\text { Proposed } \\
\text { work }\end{array}$ \\
\hline Best & 404.14 & 224.71 & 398.26 & 300.28 & 389.72 & 368.64 \\
\hline Median & 430.01 & 236.08 & 413.24 & 327.85 & 418.42 & 413.38 \\
\hline Worst & 451.10 & 260.19 & 467.87 & 368.17 & 573.48 & 498.42 \\
\hline Mean & 428.60 & 240.45 & 420.00 & 325.10 & 432.95 & 418.67 \\
\hline
\end{tabular}

Considering the total cost associated among different routes, while solving the problem at hand and the results computed, graphical representation is depicted in figure 4 and 5 . 


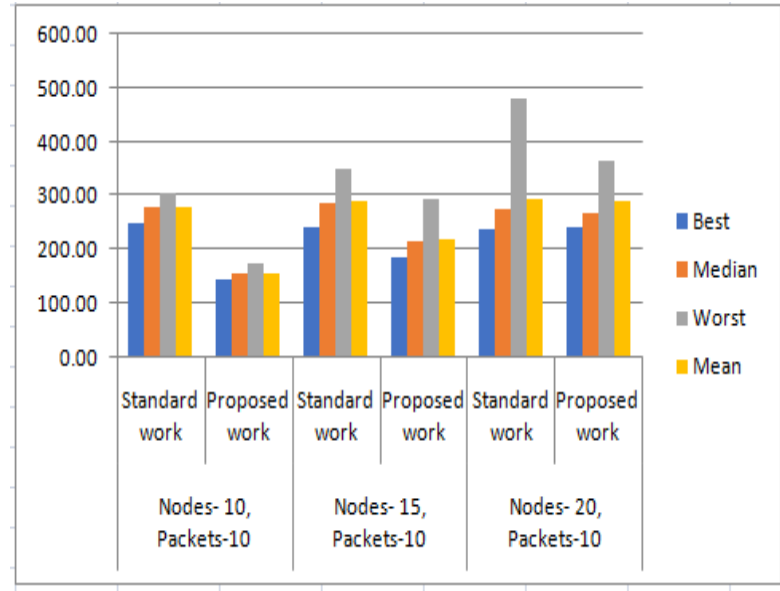

Figure 5: Graphical Representation of Comparison of Standard vs. Proposed Work, considering Cost as factor for 10 packets

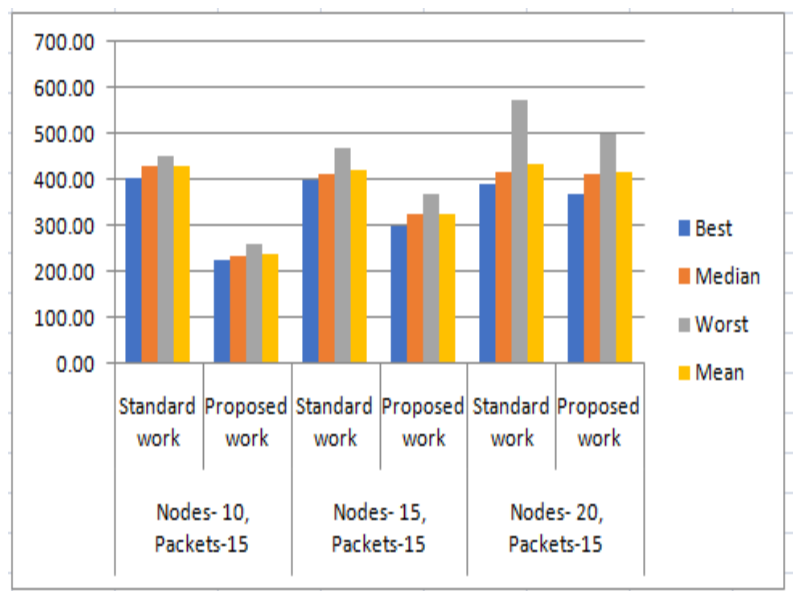

Figure 6: Graphical Representation of Comparison of Standard vs. Proposed Work, considering Cost as factor for 15 packets

Table 5: Mean Time comparison of standard bat algorithm vs. proposed algorithm

\begin{tabular}{|c|c|c|}
\hline Time (in Seconds) & Standard Work & Proposed Work \\
\hline Nodes- 10,Packets-10 & 2.32 & 2.05 \\
\hline Nodes- 10,Packets-15 & 3.43 & 3.17 \\
\hline Nodes- 15,Packets-10 & 13.16 & 12.73 \\
\hline Nodes- 15,Packets-15 & 19.57 & 19.15 \\
\hline Nodes- 20,Packets-10 & 49.70 & 46.70 \\
\hline Nodes- 20,Packets-15 & 73.36 & 72.01 \\
\hline
\end{tabular}

Table 6: Mean Cost comparison of Standard Algorithm Vs Proposed Algorithm

\begin{tabular}{|c|c|c|}
\hline Cost factor & Standard Work & Proposed Work \\
\hline Nodes- 10,Packets-10 & 280.39 & 158.36 \\
\hline Nodes- 10,Packets-15 & 428.60 & 240.45 \\
\hline Nodes- 15,Packets-10 & 288.97 & 220.34 \\
\hline Nodes- 15,Packets-15 & 420.00 & 325.10 \\
\hline Nodes- 20,Packets-10 & 293.18 & 291.08 \\
\hline Nodes- 20,Packets-15 & 432.95 & 418.67 \\
\hline
\end{tabular}

In Table 5 and 6, comparison of Standard Algorithm and Proposed Algorithm has been done on the basis of mean value of time and cost after performing the experiment 10 times for same values. The difference in mean value of both the algorithm is positive and we can say that while implementing congestion technique in proposed algorithm there is acceptable difference of time and cost.

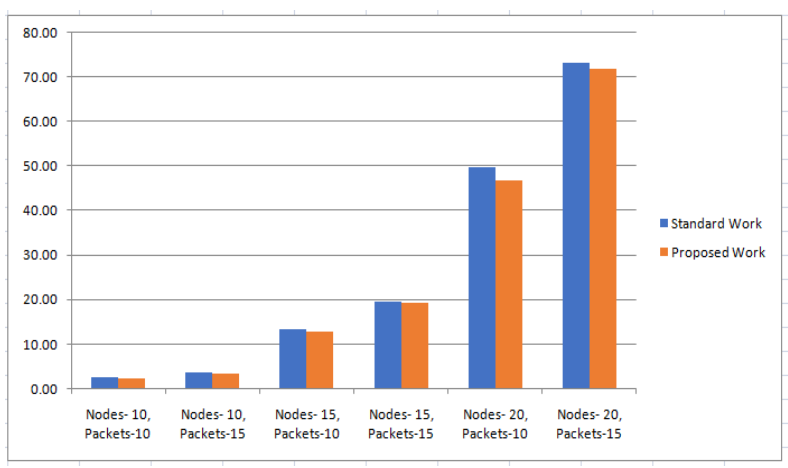

Figure 7: Comparison of Mean values of Standard and Proposed work on the basis of time factor

In figure 7 and 8, average (mean) values of time and cost of standard and proposed work are compared and represented in graphical format.

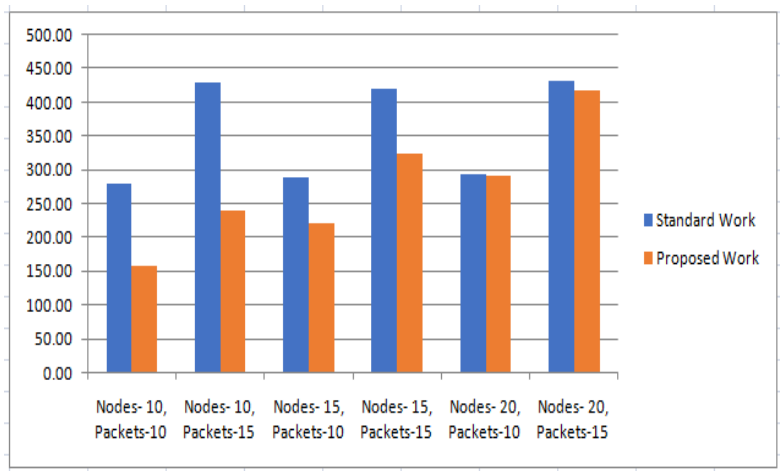

Figure 8: Comparison of Mean values of Standard and Proposed work on the basis of cost factor

\section{CONCLUSION \& FUTURE SCOPE}

Implementing infrastructure based network is not possible in different scenarios and environment, which gives rise to the development and implementation of infrastructure less networks, i.e.ad hoc network. Wireless Sensor Network is 
well known type of ad hoc network, having different aspects to work on. Routing is main aspect in wireless sensor network. Routing algorithms should be reliable and efficient in consideration to various parameters including energy consumption, congestion free, minimal packet loss, network lifetime and many more. The routing techniques used nowadays having problems of congestion occurs due to high rate of transmission on the common optimal path. Congestion causes delay in packet delivery, reduce network efficiency, consume more energy than required which are major effects on any network. Bandwidth wastage will ultimately lead to congestion, which in turn, causes the network to die.

In this paper, the main focus is to overcome the congestion problem as it causes major problems like packet loss, transmission delay and more energy consumption. This work primarily focused and found the optimal route in wireless sensor network. The experimental results are compared with [26] on the basis of best, median, worst and mean parameters for time and cost factor. The experimental results show that the proposed algorithm is cost effective, in comparison, used for solving routing problem in WSN environment. Proposed algorithm is proven to be efficient as minimizes the congestion and same time find the best route at minimal cost.

The future scope of this research work is the hybridization of bat algorithm with another nature inspired algorithm can be done to achieve better results. One can also include other parameters to develop different algorithms for achieving better and more optimal results. To extend this work, use statistical methods to decide the threshold value as the maximum limit while selecting the optimal route. Another way to decide the threshold value could be based on training and feedback mechanism of artificial neural network.

\section{REFERENCES}

[1] Bhatt, M., Sharma, S., Luhach, A. K., \& Prakash, A. (2016, September). Nature inspired route optimization in vehicular adhoc network. In Reliability, Infocom Technologies and Optimization (Trends and Future Directions)(ICRITO), 2016 5th International Conference on (pp. 447-451). IEEE.

[2] Bhatt, M., Sharma, S., Prakash, A., Pandey, U. S., \& Jyoti, K. (2016). Traffic Collision Avoidance in VANET Using Computational Intelligence. International Journal of Engineering and Technology.

[3] Jadhav, P., \& Satao, R. (2016). A survey on opportunistic routing protocols for wireless sensor networks. Procedia Computer Science, 79, 603-609.

[4] Jung, S. G., Kang, B., Yeoum, S., \& Choo, H. (2016). Trail-using ant behavior based energy-efficient routing protocol in wireless sensor networks. International Journal of Distributed Sensor Networks.

[5] Sharma, S., Luhach, A. K., \& Jyoti, K. (2016, March). Research \& analysis of advancements in BAT algorithm. In Computing for Sustainable Global Development (INDIACom), 2016 3rd International Conference on (pp. 2391-2396). IEEE.

[6] Sharma, S., Luhach, A. K., \& Jyoti, K. (2016). A Novel Approach of Load Balancing in Cloud Computing using Computational Intelligence.

[7] Shahi, B., Dahal, S., Mishra, A., Kumar, S. V., \& Kumar, C. P. (2016). A Review Over Genetic Algorithm and
Application of Wireless Network Systems. Procedia Computer Science, 78, 431-438.

[8] E. U. K. Selim Y1lmaz, "A new modification approach on bat algorithm for solving optimization problems," Applied Soft Computing, vol. 28, pp. 259-275, 2015

[9] Singh, S. P., \& Sharma, S. C. (2015). A survey on cluster based routing protocols in wireless sensor networks. Procedia computer science, 45, 687-695.

[10] C.-S. S.-F. H.-Y. L.-S. P.-T. T. Yi-Ting Chen, "A Guidable Bat Algorithm Based on Doppler Effect to Improve Solving Efficiency for Optimization Problems," Computational Collective Intelligence. Technologies and Applications, vol. 8733, pp. 373-383, 2014.

[11] F. V. C. Jonathan Pérez, "A New Bat Algorithm with Fuzzy Logic for Dynamical Parameter Adaptation and Its Applicability to Fuzzy Control Design," Fuzzy Logic Augmentation of Nature-Inspired Optimization Metaheuristics, vol. 574, pp. 65-79, 2014.

[12] H.Djelloul, "Binary bat algorithm for graph coloring problem," in Second World Conference on Complex Systems (WCCS), 2014

[13] M. T. Adis Alihodzic, "Improved Hybridized Bat Algorithm for Global Numerical Optimization," in International Conference on Computer Modelling and Simulation,IEEE, 2014.

[14] M. G. A. M. M. S. Homayun Afrabandpey, "A Novel Bat Algorithm Based on Chaos for Optimization Tasks," in Iranian Conference on Intelligent Systems (ICIS),IEEE, 2014.

[15] S. M. M. X.-S. Y. Seyedali Mirjalili, "Binary bat algorithm," Neural Computing and Applications, pp. 663681, 2014.

[16] Sharma, M. (2014, July). Wireless sensor networks: Routing protocols and security issues. In Computing, Communication and Networking Technologies (ICCCNT), 2014 International Conference on (pp. 1-5). IEEE.

[17] Topal, A. O., Altun, O., \& Terolli, E. (2014, October). Dynamic virtual bats algorithm (dvba) for minimization of supply chain cost with embedded risk. In Modelling Symposium (EMS), 2014 European (pp. 58-64). IEEE.

[18] Y. Z. Liangliang Li, "A novel complex-valued bat algorithm," Neural Computing and Applications, vol. 25, no. 6 , pp. 1369-1381, 2014.

[19] Tyagi, S., \& Kumar, N. (2013). A systematic review on clustering and routing techniques based upon LEACH protocol for wireless sensor networks. Journal of Network and Computer Applications, 36(2), 623-645.

[20] Prathap, U., Shenoy, P. D., Venugopal, K. R., \& Patnaik, L. M. (2012, December). Wireless sensor networks applications and routing protocols: survey and research challenges. In Cloud and Services Computing (ISCOS), 2012 International Symposium on (pp. 49-56). IEEE.

[21] Saleem, M., Di Caro, G. A., \& Farooq, M. (2011). Swarm intelligence based routing protocol for wireless sensor networks: Survey and future directions. Information Sciences, 181(20), 4597-4624. 
[22] Kordon, A. K. (2010). Swarm intelligence: The benefits of swarms. In Applying Computational Intelligence (pp. 145-174). Springer Berlin Heidelberg.

[23] Yang, X. S. (2010). A new metaheuristic bat-inspired algorithm. Nature inspired cooperative strategies for optimization (NICSO 2010), 65-74.

[24] Zengin, A., \& Tuncel, S. (2010). A survey on swarm intelligence based routing protocols in wireless sensor networks. International Journal of Physical Sciences, 5(14), 2118-2126.
[25] Camilo, T., Carreto, C., Silva, J. S., \& Boavida, F. (2006, September). An energy-efficient ant-based routing algorithm for wireless sensor networks. In International Workshop on Ant Colony Optimization and Swarm Intelligence (pp. 49-59). Springer Berlin Heidelberg.

[26] Manshahia, M. S., Dave, M., \& Singh, S. B. (2016). Improved Bat Algorithm Based Energy Efficient Congestion Control Scheme for Wireless Sensor Networks. Wireless Sensor Network, 8(11), 229. 